\title{
Betreutes Wohnen in Familien - Vernachlässigte Alternative zur Heimversorgung für schwer psychisch erkrankte Menschen
}

\author{
Supported Housing in Families - Alternative to Residential Care for Patients with \\ Severe Mental Illness
}

Autor

Institut
Michael Konrad

ZfP Südwürttemberg, Ravensburg

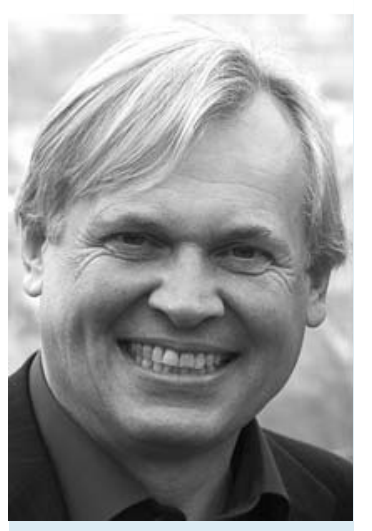

Michael Konrad

Bibliografie

DOI http://dx.doi.org/

10.1055/s-0042-109641

Psychiat Prax 2016; 43 :

239-241

(c) Georg Thieme Verlag KG

Stuttgart · New York

ISSN 0303-4259

Korrespondenzadresse

Dr. Michael Konrad

ZfP Südwürttemberg

Eisenbahnstraße 28

88212 Ravensburg

Michael.konrad@zfp-zentrum.

de
Seit der Ratifizierung der UN-Behindertenrechtskonvention durch die Bundesregierung hat sich die Diskussion über eine nicht institutionelle Wohnversorgung verstärkt. Die S3-Leitlinien Psychosoziale Therapien gehen davon aus, dass chronisch psychisch erkrankte Menschen Unterstützungsleistungen in „normalen“ Wohnungen erhalten sollen [1]. Gleichwohl stellte die UN-Kommission bei ihrer Visitation fest, dass insbesondere in Deutschland die institutionellen Wohnformen überwiegen [2]. Diese Feststellung korrespondiert mit der Tatsache, dass für nicht institutionelle Wohnformen wenig evidenzbasierte Untersuchungen vorliegen und in der letzten großen deutschsprachigen Übersicht die Frage gestellt wurde, ob Enthospitalisierung zur Entinstitutionalisierung führt [3,4].

Die Wirkung von Betreutem Wohnen wurde in erster Linie in Enthospitalisierungsstudien untersucht und hatte bei schwer beeinträchtigten Patienten einen zweifelhaften Effekt [5]. Auch im englischsprachigen Raum konnte ein positiver Effekt über RCT-Studien ausschließlich bei wohnsitzlosen Personen festgestellt werden [6]. Im gegliederten deutschen Sozialsystem scheitern entsprechende Studien bereits an der unterschiedlichen Begrifflichkeit in den verschiedenen Bundesländern und an der Tatsache, dass verschiedene Formen Betreuten Wohnens in manchen Regionen bereits eingeführt sind. Die zufällige $\mathrm{Zu}$ ordnung zu einer Wohnform ist daher aus diversen Gründen ausgeschlossen.

In der Praxis hat sich das Betreute Wohnen in ambulanter und stationärer Form unabhängig von nicht vorhandenen wissenschaftlichen Nachweisen durchgesetzt. Bei psychisch schwer beeinträchtigten Menschen werden jedoch wieder zunehmend die Grenzen einer gemeindeintegrierten Wohnversorgung diskutiert. Die Rückkehr zu den Langzeitstationen in den psychiatrischen Großkliniken ist zwar nicht mehr denkbar, aber es mehren sich insbesondere bei klinischen Psy- chiatern die Stimmen, die die Ausweitung von gut konzipierten geschlossenen Wohnheimen für unabdingbar halten [7]. Die Diskussion hat zwischenzeitlich auch die Verfechter der Gemeindepsychiatrie erfasst [8].

Ein traditionelles Versorgungsangebot der Psychiatrie bleibt in dieser Diskussion ausgespart [9]: Die Psychiatrische Familienpflege. Sie wurde in Deutschland zwar in den 1980er-Jahren wieder eingeführt [10] und firmiert zwischenzeitlich meist unter dem Titel Betreutes Wohnen in Familien (BWF) [11] und hat Ende des Jahres 2013 die Zahl von 2386 chronisch psychisch erkrankten Menschen erreicht [12]. Als Regelangebot der gemeindepsychiatrischen Versorgung existiert sie jedoch lediglich in einigen Landkreisen BadenWürttembergs und Nordrhein-Westfalens.

Dabei hatte die Familienpflege in den Fachdiskussionen politische Sprengkraft. Viele deutsche Psychiater sind an den Ursprungsort Geel gereist und sahen in Gastfamilienintegration eine Alternative zur Anstalt. Der Zwiefalter Arzt Edmund Neuschler hat seine Eindrücke nach einem Besuch in Geel im Jahr 1867 emphatisch zusammengefasst und damit der Diskussion um Asyle und Hospitalismus weit vorgegriffen: „Wer von uns, der jahrelang in einer öffentlichen Anstalt gelebt hat, war nicht oft von Mitgefühl ergriffen gegenüber den traurigen Bedingungen ihrer Insassen und den vielfältigen Einschränkungen, die sie zu ertragen haben - nicht als unvermeidliche Konsequenz ihrer Krankheit, sondern ausschließlich aufgrund des Behandlungssystems, dem sie unterworfen sind“ ([13], S.32). Die Analyse eines Erving Gofman wurde hier vorweggenommen.

Trotz vieler prominenter Befürworter wie z.B. Wilhelm Griesinger wurde die Familienpflege bei dem Aufbau der psychiatrischen Versorgung in Deutschland ignoriert. Erst als die Anstalten Anfang des 20. Jahrhunderts zunehmend überfüllt waren und der Bau neuer Anstalten aus fiskalischen Gründen eingestellt wurde, konnte sich 
die Familienpflege als Alternative zur Langzeitunterbringung in der Anstalt entfalten. In der Blütezeit am Ende der Weimarer Republik wurden über 5000 Patienten in Gastfamilien versorgt [10]. Infolge der massenhaften Ermordung psychisch kranker Menschen wurden die arbeitsfähigen Patienten aus den Gastfamilien in die Anstalten zurückgeholt, wodurch das vorläufige Ende der Familienpflege in Deutschland besiegelt war [10]. In vielen anderen Ländern blieb sie Bestandteil der psychiatrischen Versorgung. Allerdings mutierte die familiäre Integration in einigen Ländern wie der Schweiz während der Phase der Enthospitalisierung zu Familien geleiteten Kleinst- und Kleinheimen [14].

In den Studien, die Alternativen zum Hospital untersuchten, blieb die Familienpflege mit einer Ausnahme unberücksichtigt. Linn und Kollegen führten an 5 Kliniken der Veterans Administration in den USA eine Untersuchung durch und fanden heraus, dass Klienten in Gastfamilien im Follow-up nach 4 Monaten ihre sozialen Fertigkeiten und soziale Anpassung gegenüber einer Kontrollgruppe von Heimbewohnern signifikant verbessert haben [15]. Angesichts der erwähnten schwierigen Abgrenzung gegenüber Heimen war ein weiteres Ergebnis der Studie für die Beurteilung als qualitativ bedeutendes Angebot von Bedeutung. Eine deutliche Besserung war bei den Klienten festzustellen, die allein oder mit höchstens einem weiteren psychisch Kranken in einer Gastfamilie lebten [16]. Wie der Titel der Publikation besagt wurde damit die „Weisheit von Geel“ bestätigt, dass nämlich nicht mehr als 2 Klienten gleichzeitig in einer Gastfamilie leben dürfen. Damit ist auch bestätigt, dass die Familienstruktur der entscheidende Wirkfaktor im BWF ist $[17,18]$.

Das hatte bereits Griesinger erkannt: „Die familiäre Kranksinnigenpflege gewährt den Kranken das, was die prachtvollste und bestgeleitete Anstalt der Welt niemals gewähren kann, die volle Existenz unter Gesunden, die Rückkehr aus einem künstlichen und monotonen in ein natürliches, soziales Medium, die Wohltat des Familienlebens“ ([19], S.36f.) Der Begriff Wohltat hat bei der Fachwelt in der politischen Aufbruchsstimmung der PsychiatrieEnquete jedoch offensichtlich Abwehr gegenüber einem idealisierten Bild der „heilen“ Familie ausgelöst. Die Erfahrungen haben gezeigt, dass nicht die Harmonie, sondern die Aushandlung der Form des Zusammenlebens der wesentliche Aspekt des BWF ist. Die in den westlichen Industrieländern bestehende Pluralisierung der Familienformen mit der Notwendigkeit, flexibel auf die ständigen Veränderungen einzugehen [20], passt damit zu einem Krankheitsbegriff, der vom Verlust der natürlichen Selbstverständlichkeit ausgeht [21] und zu Versorgungskonzepten, die Inklusion, Diversität und Selbstbestimmung als wesentliche Merkmale der UN-Behindertenrechtskonvention in den Fokus stellen [22]. Geeignete Familien zu finden ist kein Problem: im Landkreis Ravensburg leben Menschen mit seelischer und geistiger Behinderung sowie Jugendliche mit psychischen Beeinträchtigungen derzeit in ca. 300 Gastfamilien.

Im Hinblick auf die Inklusion von Menschen mit Behinderung bietet das Betreute Wohnen in Familien ein "Alleinstellungsmerkmal«. In keinem anderen Unterstützungsangebot ist ein Integrationsprozess in das Gemeinwesen zu finden, das der Integration in eine Gastfamilie gleicht. Durch die Integration in eine solche Familie erschließt sich dem Menschen mit Behinderung gleichzeitig der Zugang zu Verwandten, Freunden, Nachbarn und Arbeitskollegen. Dass Konflikte mit der Umwelt im Gegensatz zu Erfahrungen im ambulant Betreuten Wohnen nicht zum Ausschluss führen, ist durch den Schutz der familiären Zugehörigkeit gewährleistet. Klienten in Gastfamilien fühlen sich sicherer als in anderen betreuten Wohnformen [23].
Dennoch ist davon auszugehen, dass die in einer Delphi-Studie zu Enthospitalisierungsprozessen der Universitäten Bielefeld und Erlangen gefundenen Vorurteile von Fachexperten der Psychiatrie, die keine eigenen Erfahrungen mit dem BWF haben, immer noch vorherrschen [24]:

Vorurteil 1: Die Entlohnung bewirkt eine ungünstige Auswahl. Das Gegenteil ist der Fall. Die Entlohnung bewirkt, dass Gastfamilien bereit sind, Konflikte auszutragen und damit therapeutisch wichtige Prozesse in Gang setzen [25]. Wichtig ist jedoch, dass die Entlohnung weder zu niedrig noch zu hoch ist, was natürlich von Region zu Region verschieden ist. Im ersten Fall melden sich zu wenige Familien, im zweiten Fall melden sich zu viele Familien, die von der Entlohnung abhängig sind.

Vorurteil 2: BWF ist eine Betreuungsform für wenige ausgewählte Klienten. Auch hier gilt das Gegenteil. Gastfamilien haben sich nicht nur als geeignet für Personen erwiesen, die das professionelle Versorgungssystem überfordert haben [26], sie haben sich auch bei Menschen mit Borderline-Persönlichkeitsstörung, Alkoholdemenz, Demenzkranken und bei psychisch kranken Eltern mit Kind als hilfreich erwiesen [11]. Das Potenzial wird meist dadurch begrenzt, dass der Fachdienst stets in Gefahr ist, „spezielle Spezialfamilien“ [26] von vorneherein auszuschließen.

Vorurteil 3: Entmündigung der Klienten. Dieses Vorurteil wurde von der Psychiatrieerfahrenen Sybille Prins mit dem Argument entkräftet, dass sie bei ihren Klinikaufenthalten Entmündigung eher von Pflegepersonen erfahren hätte [27].

Vorurteil 4: Schwierige Qualitätskontrolle der Gastfamilien. Das ist zutreffend. Allerdings findet sie in höherer Frequenz statt als in anderen betreuten Wohneinrichtungen durch einen begleitenden Fachdienst. Nicht in Form des Abhakens einer Checkliste, sondern als qualifizierte systemische Beratung. Der Anspruch an die Mitarbeitenden im begleitenden Fachdienst ist hoch, am besten eignen sich Fachkräfte, die längere Erfahrung in der direkten Arbeit mit schwer psychisch beeinträchtigten Menschen haben [28].

Vorurteil 5: Isolation in der Familie statt sozialer Teilhabe. Dies wurde in der vergleichenden Studie des Landschaftsverbands Westfalen-Lippe entkräftet. Demnach haben Klienten im BWF mehr Kontakte mit Nichtbehinderten, häufiger genügend Kontakt zur Nachbarschaft und gestalten die Freizeit häufiger mit Nichtbehinderten als Klienten in professionell betreuten Wohnformen [23].

Zusammengefasst kann festgestellt werden, dass im BWF die Themen Personenzentrierung und Sozialraum in einem System vereint sind. Für freiheitsliebende und gleichzeitig grenzüberschreitende Klienten ist das BWF manchmal die einzige Möglichkeit zwischen Wohnungslosigkeit und Heim. Aufgrund des Sozialisationspotentials von Familien kann BWF für sogenannte "junge Wilde» [29] ein Weg hin zu einem vollkommen selbstbestimmten Wohnen und Leben sein, der einen geeigneten Schutzraum bietet. Angesichts der geplanten gesetzlichen Änderungen, insbesondere in Bezug auf das Bundesteilhabegesetz, dürfte BWF für einen Teil schwer beeinträchtigter psychisch erkrankter Menschen die einzige personenzentrierte und die Selbstbestimmung fördernde Hilfe zur Teilhabe am gesellschaftlichen Leben sein. Während Gastfamilien in der Akutversorgung zunehmend in den Fokus genommen werden [30, 31], wird BWF für die Rehabilitation von jungen chronisch psychisch erkrankten Menschen noch relativ wenig genutzt. Die Erfahrungen in Ravensburg zeigen, dass die Unterstützung durch Gastfamilien bei jungen Klientinnen und Klienten, insbesondere mit den Diagnosen „Psychische Störungen mit Beginn im Kindes- und Jugendalter“ sowie „Bor- 
derline-Persönlichkeitsstörung“ [32], als Weg der gesellschaftlichen Inklusion geeignet ist. Diese Personengruppen bevorzugen ambulante Hilfen und sind damit im BWF bestens versorgt.

Das Fehlen von Gastfamilien im städtischen Umfeld, das in den Anfangszeiten der Wiedereinführung die Familienpflege in Deutschland charakterisiert hat [33], scheint sich so langsam aufzuheben. In anderen Ländern gibt es durchaus Erfahrungen in Großstädten, z.B. im 13. Arrondisement in Paris. Die dort gemachte Erfahrung hat gezeigt, dass es darum geht, entsprechende Gruppen von Gastfamilien zu finden. In Paris waren vor allem nordafrikanische Migrantenfamilien als Gastfamilien erfolgreich [34]. BWF sollte daher zur Standardversorgung gemeindepsychiatrischer Verbundsysteme gehören und könnte durch die universelle Nutzung das funktionale Basismodell gemeindepsychiatrischer Versorgung [35] anreichern. Ganz im Sinne des deutschen Familienpflegepioniers Gustav Kolb: „Die Familienpflege ist das beste Mittel, in einen veralteten Betrieb einen frischen Geist hineinzubringen“" 36$]$.

\section{Literatur}

1 DGPPN, Hrsg. S3-Leitlinie Psychosoziale Therapien bei schweren psychischen Erkrankungen. Heidelberg, Berlin: Springer; 2012

2 United Nations General Assembly. Thematic study on the right of persons with disability to live independently and be included in the Community. Report of the Office of the United Nations High Commissioner for Human Rights. Im Internet: www ohchr.org (Stand: 12.12.2014)

3 Bitter D, Entenfellner A, Matschnig T et al. Da-Heim im Heim!? Bedeutete Ent-Hospitalisierung auch Ent-Institutionalisierung? Psychiat Prax 2009; 36: 261 - 269

4 Bramesfeld A, Schäfer I, Stengler K et al. Stimulating mental health services research: what are the implications of the new DGPPN S3-guideline for psycho-social therapies? Psychiat Prax 2014; 41: 65-67

5 Franz M, Meyer T, Dubowy M et al. Das Akkumulieren „Neuer Langzeitbewohner" in klinikassoziierten psychiatrischen Heimen: Eine Herausforderung für die psychiatrische Versorgung. Psychiat Prax 2010; 37: $240-247$

6 Benston EA. Housing Programs for Homeless Individuals With Mental Illness: Effects on Housing and Mental Health Outcomes. Psychiatr Serv 2015; 66: 806-816

7 Kruse G. Verantwortung übernehmen für „die Schwierigsten“. Brauchen wir dazu die geschlossene Heimunterbringung? Sozialpsychiatrische Informationen 2012; 42: 14-17

8 Reumschüssel-Wienert C, Crefeld W. Geschlossene psychiatrische Wohnheime. Debatte Pro \& Kontra. Psychiat Prax 2012; 39: 4-6

9 Deister A, Wilms $B$. Innovative treatment in psychiatry - a chance for integrated health care. Psychiat Prax 2015; 42: 8-10

10 Konrad M, Schmidt-Michel P-O, Hrsg. Die zweite Familie. Psychiatrische Familienpflege. Geschichte Praxis - Forschung. Bonn: Psychiatrie-Verlag; 1993

11 Konrad M, Becker J, Eisenhut R, Hrsg. Inklusion leben. Betreutes Wohnen in Familien für Menschen mit Behinderung. Freiburg: Lambertus; 2012

12 Fachausschuss BWF. Anzahl der Vermittlungen in der BRD 2013. Im Internet: www.bwf-info.de (Stand: November 2013)

13 Neuschler E. A visit to Geel. A letter to the editors of the Journal of Mental Science; translated with remarks by John Sibbald. J Ment Sc 1867; 13: $20-43$

14 Uchtenhagen A. Familienpflege. In: Frießem DH, Hrsg. Kritische Stichwörter zur Sozialpsychiatrie. München: Fink; 1979: 297-302

15 Linn MW, Caffey EM, Hogarty G et al. Hospital vs. community (foster) care for psychiatric patients. Arch Gen Psychiatry 1977; 34: 78-83

16 Linn MW, Klett CJ, Caffey EM. Foster home characteristics and psychiatric patient outcome. The wisdom of Gheel confirmed. Arch Gen Psychiatry 1980; 37 : $129-132$
17 Konrad M. Die Familiengeschichte der Gastfamilie als milieutherapeutischer Faktor in der psychiatrischen Familienpflege (unveröffentlichte Dissertation). Ulm: Universität Ulm; 1992

18 Schönberger C. Die diskrete Arbeit der Transformation. Soziologische Fallstudien zum Leben psychisch kranker Menschen in Fremdfamilien. Bern: Huber; 2007

19 Griesinger W. Über Irrenanstalten und deren Weiterentwicklung in Deutschland. Arch Psych 1868; 1: 8-43

20 Keupp H. Familie ist auch nicht das was sie einmal war. Von der selbstverständlichen Matrix zum Balanceakt. In: Konrad M, Becker J, Eisenhut R, Hrsg. Inklusion leben. Betreutes Wohnen in Familien für Menschen mit Behinderung. Freiburg: Lambertus; 2012: 47-58

21 Bock T, Heinz R. Psychosen. Ringen um Selbstverständlichkeit. Köln: Psychiatrie-Verlag; 2016

22 Konrad M. Inklusion - ein Ausweg zwischen Heim und ambulantem Ghetto. Sozialpsychiatrische Informationen 2015; 45: 31 - 36

23 Richter D. Evaluation des stationären und ambulant betreuten Wohnens psychisch behinderter Menschen in den Wohnverbünden des Landschaftsverbands Westfalen-Lippe. Psychiat Prax 2010; 37: 127 133

24 Ingenleuf HJ. Das Ende des Orakelns: Antworten auf die Kritik an der psychiatrischen Familienpflege. Sozialpsychiatrische Informationen 2004; 34: 39-45

25 Krüger $M$, Konrad $M$, Schmidt-Michel P-O. Ist die Integration chronisch psychisch Kranker in Gastfamilien in der modernen Gesellschaft möglich? MMG 1988; 13: 11-20

26 Neuenfeldt-Spickermann R. Die gute Familie. Aspekte zur Auswahl geeigneter Gastfamilien. In: Konrad M, Becker J, Eisenhut R, Hrsg. Inklusion leben. Betreutes Wohnen in Familien für Menschen mit Behinderung. Freiburg: Lambertus; 2012: 148-158

27 Prins S. Betreutes Wohnen in Familien - Chancen und Probleme. Die Sicht einer Psychiatrie-Erfahrenen. In: Konrad M, Becker J, Eisenhut R, Hrsg. Inklusion leben. Betreutes Wohnen in Familien für Menschen mit Behinderung. Freiburg: Lambertus; 2012: 69-74

28 Wermuth K, Konrad M, Eisenhut R, von Bebenburg M. Zu dritt! Die Funktion des Begleitenden Fachdienstes im Betreuten Wohnen in Familien. In: Konrad M, Becker J, Eisenhut R, Hrsg. Inklusion leben. Betreutes Wohnen in Familien für Menschen mit Behinderung. Freiburg: Lambertus; 2012: 113 - 133

29 Huck W. Wahnsinnig jung. Junge Erwachsene zwischen Pädagogik und Psychiatrie. Köln: Psychiatrie-Verlag; 2015

30 Lötscher K, Bridler R. Psychiatrische Akutbehandlung in Gastfamilien. In: Rössler W, Kawohl W, Hrsg. Soziale Psychiatrie - Band 1. Stuttgart: Thieme; 2013: 255-264

31 Heissler M. Was wäre, wenn die Psychiatrie-Enquete in den Kliniken tatsächlich zu einer Praxis der Deinstitutionalisierung geführt hätte? In: Armbruster J, Dieterich A, Hahn D, Ratzke K. 40 Jahre PsychiatrieEnquete. Blick zurück nach vorn. Köln: Psychiatrie-Verlag; 2015: $199-214$

32 Matt K, Röscheisen C. Menschen mit Borderline-Störung in Gastfamilien. In: Konrad M, Becker J, Eisenhut R, Hrsg. Inklusion leben. Betreutes Wohnen in Familien für Menschen mit Behinderung. Freiburg: Lambertus; 2012: 113-133

33 Konrad M, Schmidt-Michel P-O. Rückfall in die Steinzeit? Eine Zwischenbilanz 20 Jahre nach der Wiederentdeckung der Psychiatrischen Familienpflege in Deutschland. Sozialpsychiatrische Informationen 2004; 34: $2-6$

34 Held T. Psychiatrische Familienpflege. Ergebnisse einer prospektiven elfjährigen Langzeitstudie. Stuttgart: Enke; 1989

35 Steinhart I, Wienberg G. Das Funktionale Basismodell für die gemeindepsychiatrische Versorgung schwer psychisch kranker Menschen Mindeststandard für Behandlung und Teilhabe. Psychiat Prax 2016; 43: $65-68$

36 Kolb G. Die Familienpflege unter besonderer Berücksichtigung der bayrischen Verhältnisse. Z Ges Neurol Psychiatr 1911; 6: 273-304 